\title{
Chondroblastoma of the Temporal Bone: A Case Report and Literature Review
}

\author{
Katarína Obtulovičováa, ${ }^{1,}$, Marián Sičák ${ }^{1}$, Adrian Kališ², ${ }^{2}$, Tomáš Buday ${ }^{4}$
}

\begin{abstract}
Introduction: Temporal bone chondroblastoma is a rare, locally aggressive tumour originating from immature cartilage, which recurs to a high degree. Treatment is surgical. Radiotherapy is reserved for recurrence. We describe a case of a 15-year-old-boy choosing a conservative surgical approach with reconstruction of the posterior canal wall. This study aims to report a rare pediatric case. Methods: A literature review was performed to better understand temporal bone chondroblastomas, to describe their histopathological and radiological characteristics and to establish the optimal surgical and non-surgical treatments. The research of previous published data was done using PubMed with keywords mentioned below.

Results: Authors present a case of a 15-year-old boy with hearing impairment and facial nerve palsy. Conservative surgery with reconstruction of the tympanic membrane and posterior wall of the external auditory canal, restoring the hearing has been performed. We did not administer any adjuvant therapies. No sign of recurrence was observed 1 year after primary surgery. Facial nerve function is normal, and hearing is satisfactory.

Conclusion: Chondroblastomas account for less than $1 \%$ of primary bone tumours. Temporal bone chondroblastoma is rare, locally aggressive, with a high prevalence of recurrence. This study describes specific histopathological and radiological findings, the chosen surgical approach and follow-up to improve the management and the prognosis of patients affected with this particular clinical entity.
\end{abstract}

\section{KEYWORDS}

chondroblastoma; temporal bone tumour; facial nerve palsy

\section{AUTHOR AFFILIATIONS}

${ }^{1}$ Otorinolaryngology/Head \& Neck Surgery Clinic, the Central Military Hospital - Faculty Hospital, Ružomberok, Slovakia

${ }^{2}$ Institute of Pathology, the Central Military Hospital - Faculty Hospital, Ružomberok, Slovakia

${ }^{3}$ Catholic University in Ružomberok, Slovakia

${ }^{4}$ The Jessenius Medical Faculty of Comenius University in Martin, Slovakia

* Corresponding author: Otolaryngology/Head \& Neck Surgery Clinic, Central Military Hospital - Faculty Hospital, Generála M. Vesela 21, 03426 Ružomberok, Slovakia, e-mail: obtulovicovak@gmail.com 


\section{INTRODUCTION}

Temporal bone tumours represent a heterogeneous group of tumours with different biological behaviours. The specificity of this site represents a challenging surgical approach and often risk resection bringing postoperative morbidity. Histopathological findings, grading and staging in the case of malignant tumours determine the possibilities of non-surgical cancer therapy.

\section{CASE REPORT}

The ENT outpatient office is visited by a 15-year-old healthy boy due to buzz in his right ear since early April 2018. He had never had trouble with his ears before. At the initial examination, cerumen was partially removed, accompanied by haemorrhage from the ear canal. Ear drops, and systemic antibiotics were prescribed to the patient. During the check-up visit, otitis externa granulomatosa was diagnosed and the patient was sent to the clinic for further diagnosis and treatment. On April 18, 2018, an outpatient biopsy was collected from the granulation tissue of the ear canal with histopathological findings of nonspecific granulation tissue, suspected of cholesteatoma. In the microbiological examination, Staphylococcus aureus and Escherichia coli were found. Audiometry, a slight conductive hearing loss with the air-bone gap of up to $20 \mathrm{~dB}$ on the right ear was diagnosed. From April 21, 2018, paresis of facial nerve of grade IV of House - Brackmann classification developed. An HRCT scan of the temporal bone was conducted with the finding of expansive intraosseal soft tissue with calcifications in the temporal bone to the right (Fig. 1). We proceeded with surgical treatment without delay. On April 27, 2018, temporal bone tumour resection with neuromonitoring of the facial nerve on the right in the range of canal-wall down mastoidectomy with retrofacial tympanotomy (modified Fisch A approach) and subsequent reconstruction was performed. A sample was

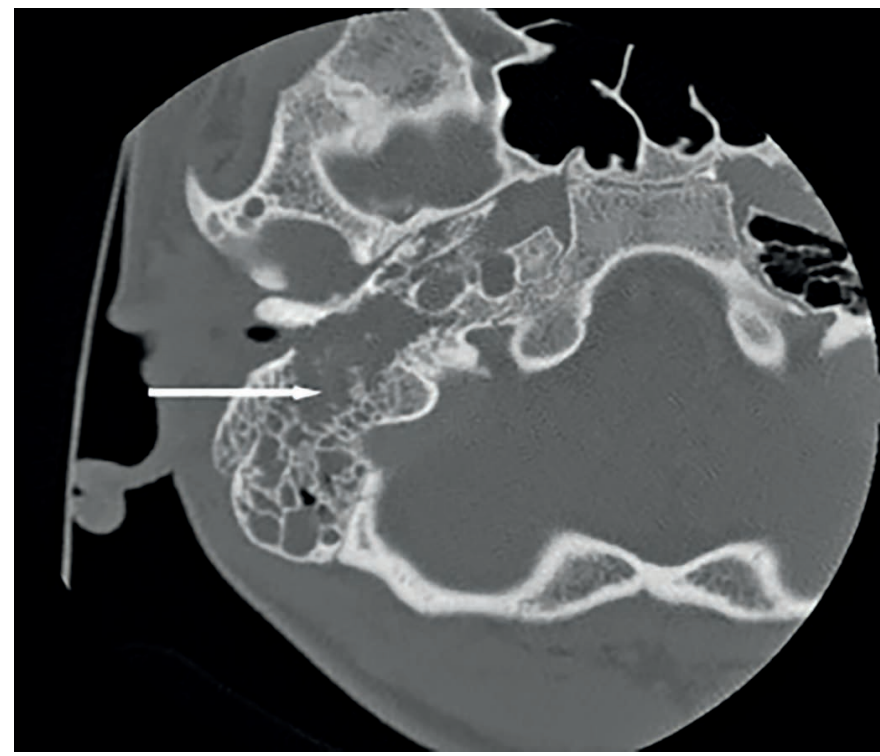

Fig. 1 A preoperative HRCT scan of the temporal bone with an expansive intraoseeal soft tissue with calcifications (white arrow). sent for perioperative histology with the conclusion - mesenchymal malignant tumour.

The tumour tissue eroded the posterior wall of the ear canal - tumour masses filled the tympanic cavity and grew to the mastoid portion of the facial nerve (Fig. 2). The tympanic membrane was largely destructed, while the chain of ossicles remained complete but surrounded by tumorous mass. We removed the incus. We left the malleus and stapes in situ. The integrity of the facial nerve was intact. The jugular bulb was intact, the tumour did not grow from it. The ACI channel was intact (Fig. 3). We reconstructed the tympanic membrane and covered the cavity that arose in the hypotympanic area after drilling the bone by a cartilage graft. The facial nerve was covered by cartilage and muscle lobe. We have planned a second-look operation.

After definitive processing, the histopathological diagnosis of chondroblastoma was determined - a tumorous

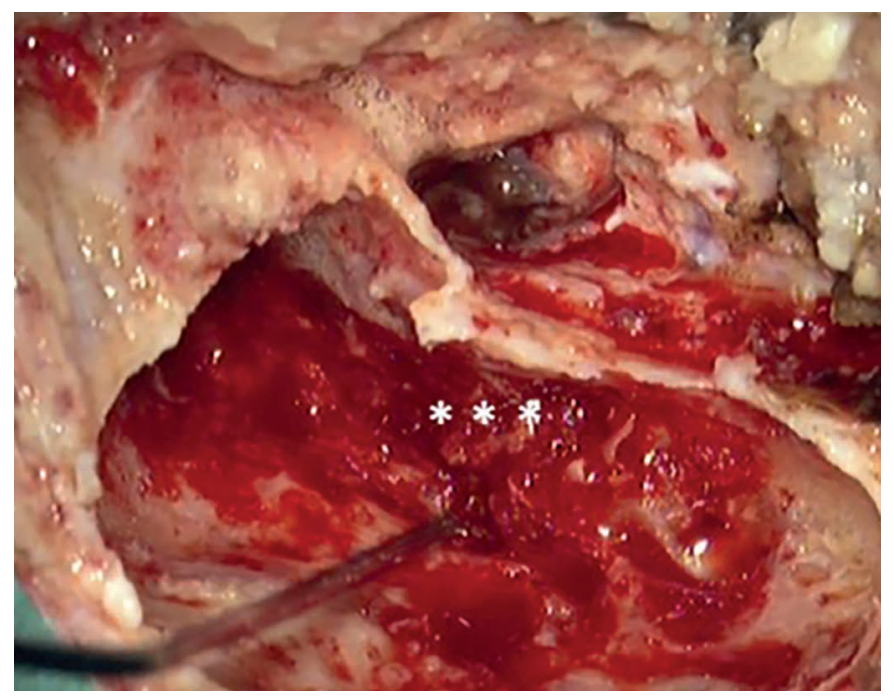

Fig. 2 A perioperative shot from primary surgery: evident destruction of the media third of the external ear canal (***). The neuromonitoring probe is attached to the mastoid region CN VII, right side. (NIM neuro 3 fi Medtronic).

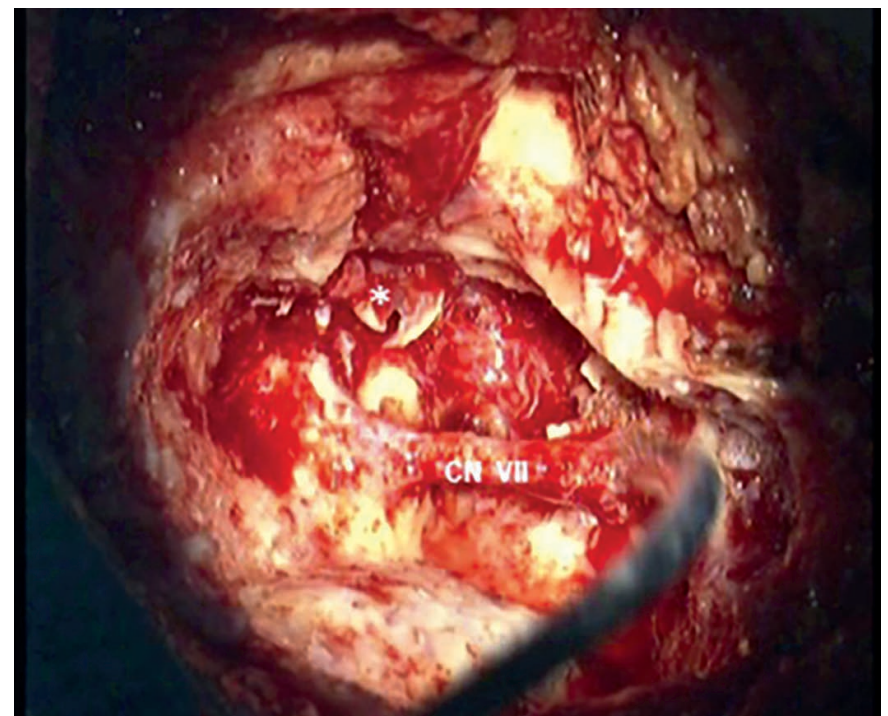

Fig. 3 A perioperative shot from primary surgery: after tumour remediation ( ${ }^{*}$ tympanic membrane). Completely denuded mastoid region CN VII, right side. (modified Fisch A). 
lesion of the chondroosteoid appearance with round uniform $\mathrm{S} 100+$ cells, with the presence of gigantic multinuclear cells (CD68+).

After the surgery, the facial nerve function rapidly adjusted, with a slight conduction disorder with the air-bone gap of up to15dB.

On June 15, 2018, we carried out a control biopsy from granulation of the auditory canal, which was histologically negative. After complete healing, we found a favourable otomicroscopic finding - a solid ear canal without dehiscence and a solid neomyrinx in good standing.

In a post-operative follow-up an FDG-PET scan was performed on June 25, 2018, with the result being a slight focal elevation of FDG metabolism in the post-operative changes field to the right together with the recommendation of the dynamics follow-up due to a short time period since surgery (Fig. 5). In other localities, we did not find pathological lesions with increased FDG metabolism of the characteristics of tumour tissue.

In the further follow-up, MRI of the temporal bone was performed on July 10, 2018, and February 4, 2019, without finding a tumour recurrence.

On March 13, 2019, we performed a planned second-look operation with control biopsies, preceded by HRCT of the temporal bone - suspected residual tumour not confirmed (Fig. 4).

The original trepanation cavity was revised - samples were taken for perioperative histology - without tumour finding, as confirmed by definitive histology.

The trepanation cavity was freely ventilated through aditus ad antrum and retrofacial tympanotomy. Stimulation of CN VII, was functional. The in situ malleus and stapes in soft contact with the neomyrinx. Between the stapes and the neomyrinx, we inserted cartilage interposites. The original reconstruction of the tympanic membrane and the ear canal from the previous operation was preserved.

The patient remains without recurrence 2 years after surgery, in the dispensary care of our otologic outpatient office and the paediatric oncology outpatient office.

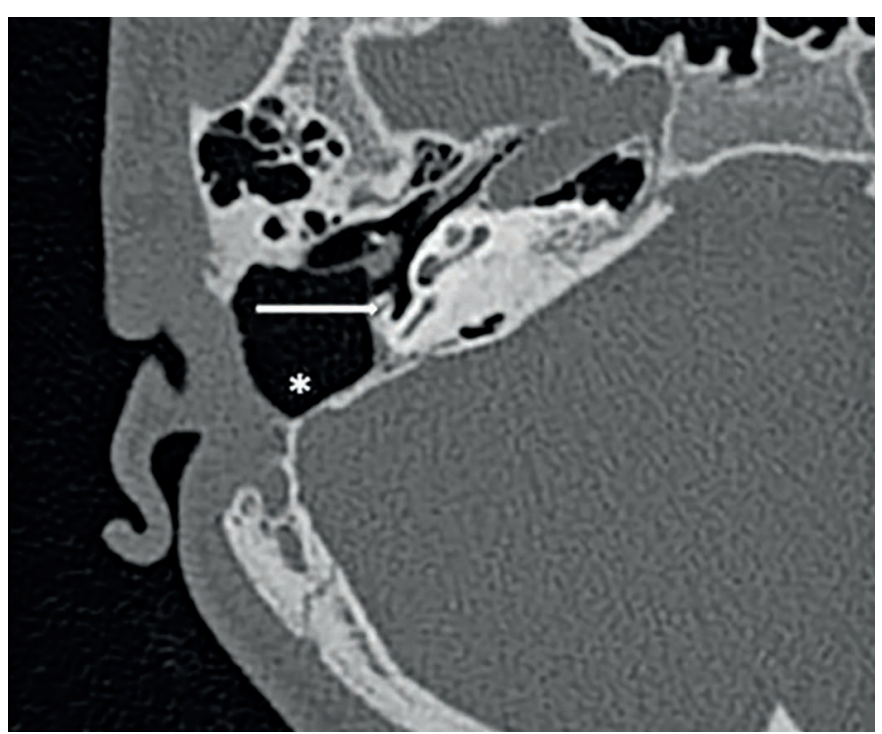

Fig. 4 A postoperative HR CT scan: the ventilated cavity of temporal bone $\left({ }^{*}\right)$ after chondroblastoma remediation without recurrence finding (CN VII - white arrow).

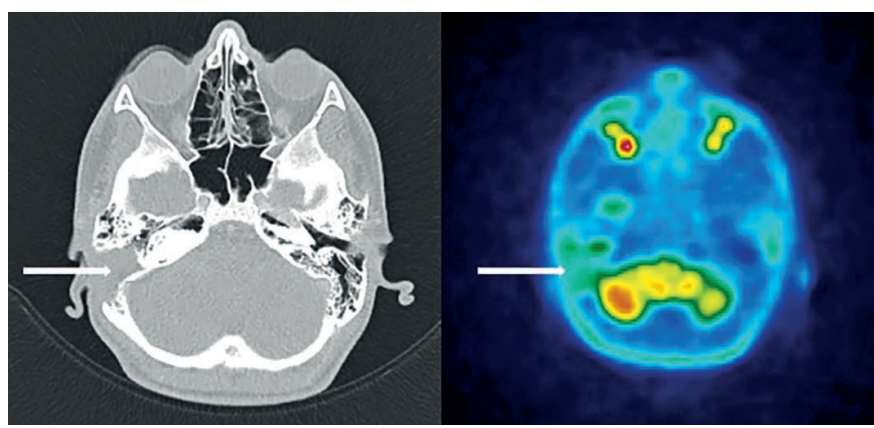

Fig. 5 A postoperative PET-CT scan of non-specific finding (white arrow) of the temporal bone after chondroblastoma remediation without recurrence finding.

\section{DISCUSSION}

Chondroblastomas represent less than $1 \%$ of primary bone tumours (1). The phenomenon was first described by Codman in 1931 as an "epiphyseal chondromatous large cell tumour of the proximal humerus". The term chondroblastoma was first used by Jaffe and Lichtenstein in 1942 (2).

It is a benign, cartilage-producing tumour most commonly occurring in the epiphyses of growing patients. A typical location is the knee, rib and pelvis. Most patients are 10-15 years old with male predominance. In the temporal bone, chondroblastoma is very rare. Patients with skull and temporal bone disease are older (40-50 years). It is a locally aggressive tumour (3).

From a histopathological point of view, chondroblasto$\mathrm{ma}$ is a tumour from immature cartilage. It is a biological intermediate between chondroma and chondrosarcoma. Its biological behaviour ranks among low-grade tumours (4). Haematoxylin-eosin staining is characterized by cartilage with immature cells. The diagnosis of chondroblastoma is inevitably complemented by the positivity of CD-68, vimentin and S-100 protein (1).

In the preoperative diagnosis, there is a clear place for HRCT in the temporal bone and MRI imaging. Investigations will allow you to accurately determine the extent of disability and the choice of optimal surgery.

Typically, on HRCT, the tumour is shown as an expansive intraoseeal soft tissue with calcifications and postcontrast enhancement sites. Marginal and central calcifications are visible (Fig. 1). In a radiological study of 16 chondroblastoma patients Park found an invaded squamous portion of the temporal bone, temporal and infratemporal pits, temporomandibular joint, and the tympanic cavity (5).

In MRI imaging, chondroblastoma manifests itself as an expansive heterogeneous hypermetabolic mass with 2 distinct components - solid with predominantly low sig$\mathrm{nal}$ in $\mathrm{T} 1$ and $\mathrm{T} 2$ sequences and multilocular cystic with $\mathrm{T} 1$ and $\mathrm{T} 2$ elongation and presence of fluid-fluid signals at T2 imaging. Post-contrast saturation is present in the solid component and septum of the cystic component. Possible high signalling in T2 imaging depends on potential haemorrhage in the tumour mass. MRI better defines the infiltration of the dura, cerebral, intracranial, and soft tissue infiltration. There was no observed increase in the signal strength for DWI $(5,6)$. 
In an FDG-PET examination, chondroblastoma is shown as a hypermetabolic lesion with strong uptake of deoxyglucose. Typically, there is increased FDG uptake, which increases from early to late phase. This type of dynamic increase in FDG uptake should lead to suspicion of bone tumour of the temporal bone (7). FDG-PET enables the identification of metastases, precise staging and diagnosis of tumour recurrence.

The treatment of choice is complete multidisciplinary tumour removal depending on the extent (3). In surgical resection, a conservative approach is recommended with a reduction of postoperative morbidity (2).

$80-90 \%$ of chondroblastomas are treated with surgery. Local recurrence occurs between $14-18 \%$ and is often within two years. Temporal bone lesions have a higher incidence of recurrence (up to 50\%) due to anatomical localization and difficult surgical extirpation (3). The role of radiotherapy is not clearly defined according to the current knowledge. Due to the low-grade character of the tumour and the absence of metastases, the postoperative radiotherapy is not indicated $(2,8)$.

The existence of a pure malignant variant of chondroblastoma is controversial. Mostly, it is a case of post-radiation sarcoma and misdiagnosis (3). The indication for radiotherapy is a recurrence. Chemotherapy is not indicated.

Reid reported a literary review of 81 cases of chondroblastoma of the temporal bone from 1950 to 2011. It gives a predilection of the incidence in men $(1: 1.2)$. The mean age of diagnosis was 41 years, with age ranges from 3 to 85 years. The symptomatology was very diverse, and its analysis did not prove a clear clinical picture that would lead to the diagnosis of chondroblastoma. Impaired hearing dominated (49.4\%), followed by cranial nerve involvement (43.2\%), facial swelling (22.2\%), otalgia (19.8\%), full ear feeling $(14.8 \%)$, otorrhea (8.6\%), tinnitus, temporomandibular pain, trismus and cephalea (2).

Reid et al. were able to evaluate follow-up in 61 (out of 81 ) patients. He was diagnosed with a recurrence in $8.2 \%$, with an average time to recurrence of 12.9 months. All five patients diagnosed with recurrence have undergone surgical treatment and three postoperative radiotherapies (2).

Selesnick et al. analysing 21 patients with chondroblastoma of the temporal bone from the 1966-1998 literature review found $95 \%$ invasion of the middle cranial pit and $76 \%$ found erosion of the external auditory canal. It hypothesizes that chondroblastoma of the temporal bone may be the result of growth from the embryonic or cartilage base in the tympanosquamous suture of the middle cranial pit. It is a separate entity (8).

Adnot et al. report on the case of chondroblastoma, which manifested itself as a preauricular mass with temporal bone and temporomandibular joint invasion. Complete remission 4 years after surgical treatment has been reported (9).

Hatano et al. report on a 67-year-old female patient with right-sided mixed hearing impairment, finding granulation in the external ear canal, feeling full in the ear, otalgia and bloody otorrhea, and pain in the temporomandibular joint. Chondroblastoma with temporomandibular joint invasion, pterygopalatine fossa and infratemporal fossa were diagnosed in the patient. She underwent resection via infratemporal approach $B$, with a facial nerve function preservation. 7.5 years after surgery, there is no sign of recurrence (10).

To date, no case of temporal bone chondroblastoma with metastasis has been reported in the literature. In contrast, metastases in the abdomen and in the lungs have been described in pelvic chondroblastoma.

With adequate surgery, the prognosis is good. Longterm monitoring of the patient using imaging is recommended.

\section{SUMMARY}

Temporal bone chondroblastoma is a rare, locally aggressive tumour originating from immature cartilage, which recurs to a high degree. The diagnosis is based on detailed imaging and histopathological examination. Treatment is surgical. Its low-grade character makes it possible to select an operative performance in which the tumour is completely resected and, at the same time, a conservative approach with a reduction in postoperative morbidity. $\mathrm{Ra}-$ diotherapy is reserved for recurrence. Long-term patient follow-up using imaging examinations is indicated.

\section{ACKNOWLEDGEMENTS}

Mrs. Jana Plevková is acknowledged for her technical assistance.

\section{FINANCIAL DISCLOSURE INFORMATION}

None.

\section{REFERENCES}

1. Cotran R, Kumar V, Robbins S. Pathological basis of disease. Fifth edition. Pennsylvania: WB Saunders Company, 1994.

2. Reid LB, Wong DS, Lyons B. Chondroblastoma of the temporal bone: a case series, review and suggested management strategy. Skull Base Rep 2011; 1(2): 71-82.

3. Fletcher CDM, Bridge JA, Hogendoorn P, et al. WHO Classification of Tumours of soft tissue and bone. Fourth edition. Lyon: IARC Press, 2013.

4. Cabrera RA, Almeida M, Mendonca ME, Frable WJ. Diagnostic pitfalls in fine-needle aspiration cytology of temporomandibular chondroblastoma: report of two cases. Diagn Cytopathol 2006; 34(6): 424-9.

5. Park SW, Kim, JH, Park JH, et al. Temporal bone chondroblastoma: imaging characteristics with pathologic correlation. Head Neck 2017; 39(11): 2171-9.

6. Kobayashi Y, Murakami R, Toba M, et al. Chondroblastoma of the temporal bone. Skeletal Radiol 2001; 30(12): 714-8.

7. Toriihara A, Tsunoda A, Takemoto A, et al. Dual-time-point FDGPET/CT Imaging of Temporal Bone Chondroblastoma: A report of two cases. Asia Ocean J Nucl Med Biol 2015; 3(2): 120-4.

8. Selesnick SH, Levine JM. Chondroblastoma of the temporal bone. Consistent middle fossa involvement. Skull Base Surg 1999; 9(4): 301-5.

9. Adnot J, Langlois, O, Tollard E, et al. Lateral skull base chondroblastoma resected with facial nerve posterior transposition. Neurochirurgie 2017; 63(2): 88-90.

10. Hatano M, De Donato G, Falcioni M, Sanna M. Chondroblastoma of the temporal bone. Acta Otolaryngol 2011; 131(8): 890-5. 